\title{
Reconstructing Neuronal Signaling Pathways With the Potential for Disruption in Schizophrenia
}

\author{
Nicole Ezell \\ Reed College Department of Biology \\ 3203 SE Woodstock Blvd \\ Portland, OR \\ nezell@alumni.reed.edu
}

\author{
Anna Ritz \\ Reed College Department of Biology \\ 3203 SE Woodstock Blvd \\ Portland, OR \\ aritz@reed.edu
}

\begin{abstract}
Schizophrenia affects about $1 \%$ of the population. It is poorly characterized. A pathway model of disruption offers a good tool to observe network-wide disruptions. Here, we use PathLinker to link hand-curated receptors and transcription factors, and combine the resulting networks into metanetworks. Topologically, schizophrenia pathways were found to not have significantly more node overlap than a random sampling, but were found to have significantly more edge overlap $(\mathrm{p}=0.0661, \mathrm{p}<0.0001)$. PathLinker reconstructed well-known signaling proteins, proteins known to be associated with schizophrenia, and neuronal proteins not previously studied in a schizophrenia context.
\end{abstract}

\section{Keywords}

cell signaling; pathway analysis; schizophrenia

\section{INTRODUCTION}

Schizophrenia is a highly heterogeneous and poorly characterized disorder. In heterogeneous diseases, genetic risk is passed down through a large number of alleles, ranging from common, small effect alleles to rare de novo mutations with large effects, which are very difficult to discover using a genomic approach.

Genomic studies have yielded several genes that contribute to the heritable risk of schizophrenia [2]. However, they are limited by the "long tail" phenomenon, which statistically makes the discovery of rare but collectively frequent mutations difficult. Instead, a pathway analysis could be used to more accurately model the disruption of physiological processes in schizophrenia.

Neurons, like all cells, communicate through a process called signal transduction. Cell-cell signaling typically begins at a receptor, and proceeds through a series of protein interactions until it reaches a transcription factor, which can activate or deactivate a particular gene's expression. This can include dozens to hundreds of proteins.

Permission to make digital or hard copies of all or part of this work for personal or classroom use is granted without fee provided that copies are not made or distributed for profit or commercial advantage and that copies bear this notice and the full citation on the first page. Copyrights for components of this work owned by others than ACM must be honored. Abstracting with credit is permitted. To copy otherwise, or republish, to post on servers or to redistribute to lists, requires prior specific permission and/or a fee. Request permissions from permissions@ acm.org.

$B C B$ '16 October 02-05, 2016, Seattle, WA, USA

(C) 2016 ACM. ISBN 978-1-4503-4225-4/16/10 . \$ $\$ 15.00$

DOI: http://dx.doi.org/10.1145/2975167.2985662
A pathway model of disruption offers another tool to uncovering the risk architecture of schizophrenia. Instead of looking at single-event mutations, pathway models look at protein-protein interaction networks. This method can be used to synthesize a large amount of heterogeneous data into a unified structure, observing where disruptions might occur and what their downstream effects may be.

We use PathLinker, an algorithm that reconstructs signaling pathways from protein-protein interactomes, to build signaling pathways potentially disrupted in schizophrenia. We input receptors and transcription factors known to be associated with schizophrenia, and analyze the topology of the resulting networks using node overlap percentages. From here, we create metanetworks based on distance, and qualitatively observe the resulting proteins forming the paths between chosen receptors and transcription factors.

\section{METHODS}

\section{PathLinker.}

We are given a directed graph $G=(V, E)$ describing an interactome, where nodes represent proteins and edges represent interactomes among proteins. The interactome $G$ has an associated cost for each edge $e \in E$ [3]. Given a source set of nodes $S \subseteq V$ and a target set of nodes $T \subseteq V$, Pathlinker efficiently calculates the $k$ shortest paths from any source node in $S$ to any target node in $T$ [4].

In this work, we run PathLinker using iterative pairs of targets and receptors; i.e., for every receptor in the source set $S=s_{1}, s_{2}, \ldots s_{x}$ and every transcription factor in the target set $T=t_{1}, t_{2}, \ldots t_{y}$, PathLinker computed the $k=100$ paths between each possible pair $\left(s_{x}, t_{y}\right)$.

\section{Datasets.}

Sources and targets were hand-curated from the literature. Receptors were chosen as sources if they were significantly associated with schizophrenia etiology, either directly through GWAS significance or gene expression data, or indirectly due to association with a neurotransmitter whose metabolism was known to be disrupted in schizophrenia. Receptors that are known drug targets were also selected, namely, D2 and nicotinic acetylcholine receptors. Additionally, the receptor list was expanded to include receptor families, most or all subunits of receptors, and select isoforms, in order to broaden the possible interactions in the incomplete interactome. This yielded 48 receptors.

Five transcription factors were similarly chosen from the literature, either because they were significantly associated 
in the GWAS or were known to regulate a gene that was significantly associated with schizophrenia [2].

We use the same interactome $G$ as is described in [4], comprised from numerous protein-protein interaction and signaling databases.

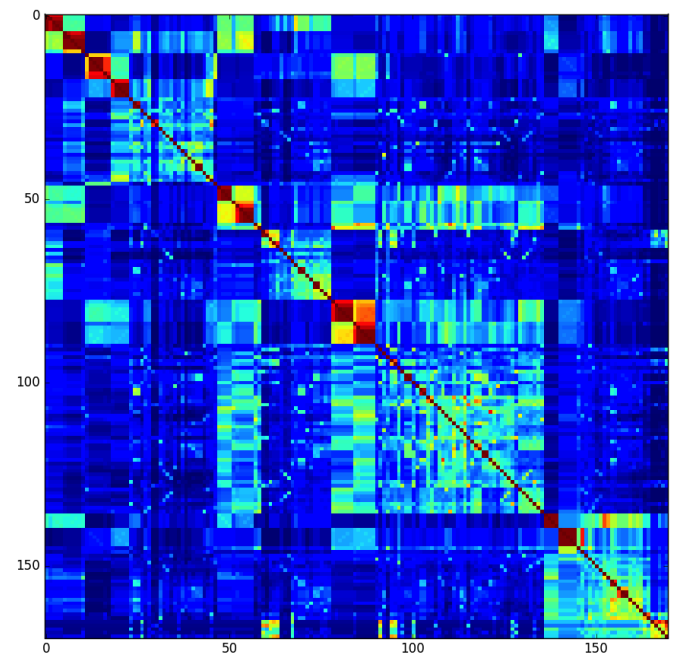

Figure 1: Heatmap showing node overlaps of 170 networks calculated from hand-curated receptors and transcription factors associated with schizophrenia. Warmer colors denote high node overlap.

\section{RESULTS}

From the $48 \times 5$ possible pairs of receptors and transcription factors, PathLinker generated 170 networks of 100 paths each. Several sources were absent from the interactome.

We calculated the node overlap between all pairs of the 170 networks and clustered them using the scipy hierarchical clustering package in Python (Figure 1). We find that subsets of networks have similar nodes, despite different receptor/transcription factor pair. We combined each cluster of networks with a similarity score of 2 or better into metanetworks and visualized these metanetworks in an interactive graph visualization tool called GraphSpace.

PathLinker reconstructed several well-known signaling proteins, including G-coupled proteins and MAPK complexes. Several key proteins from the resulting pathways were then analyzed through a literature search. Several proteins had published literature linking them to schizophrenia literature, and others had no published literature that showed that they had been studied in a schizophrenia context, making them good candidates for future study.

For example, Figure 2 shows GNG, GNA, and GNB families, which are all G-protein coupled receptors, well-known to be involved in signaling. PIK3R1 is known to be implicated in bipolar disorder and schizophrenia [1], and ABL1 has not been studied in a schizophrenia context, but has been implicated with neuronal death and inflammation in mice forebrains [5].

Finally, we compared the heatmaps to PathLinker results on a random sample of receptor/transcription factor pairs. Schizophrenia pathways were found to not have significantly more node overlap than a random sampling $(\mathrm{p}=0.0661$, student's t-test), but were found to have significantly more edge

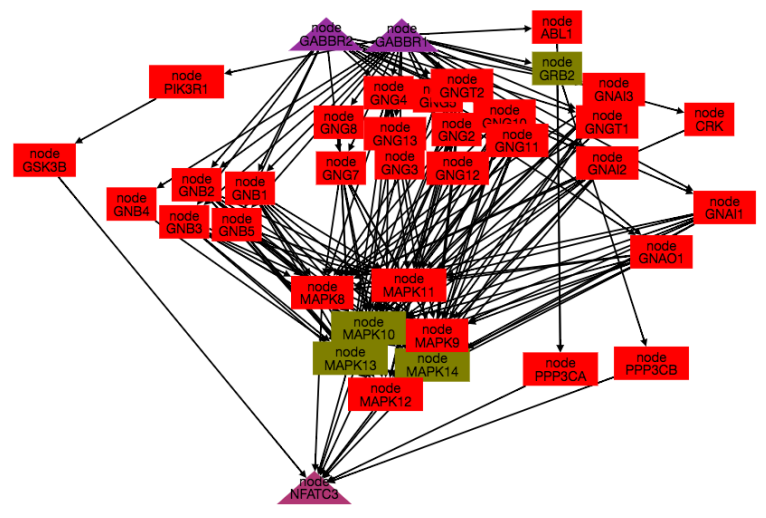

Figure 2: Metanetwork that combines two of the 170 networks, including GABBR1 and GABBR2 receptors connected to NFATC3 transcription factor. Purple triangles are from the hand-curated dataset. Red nodes are in both datasets, and brown nodes are only present in one.

overlap ( $\mathrm{p}<0.0001$, student's t-test).

\section{CONCLUSIONS}

Network analysis offers a powerful tool to use in conjunction with genomic results. This method successfully reconstructs well-known signaling pathways, proteins known to be involved in schizophrenia etiology, and offers good hypotheses for future molecular physiology studies.

\section{REFERENCES}

[1] C. Chen, C. Zhang, L. Cheng, J. L. Reilly, J. R. Bishop, J. A. Sweeney, H.-Y. Chen, E. S. Gershon, and C. Liu. Correlation between DNA methylation and gene expression in the brains of patients with bipolar disorder and schizophrenia. Bipolar disorders, 16(8):790-9, dec 2014.

[2] S. W. G. o. t. P. G. Consortium. Biological insights from 108 schizophrenia-associated genetic loci. Nature, 511(7510):421-427, jul 2014.

[3] C. L. Poirel, R. R. Rodrigues, K. C. Chen, J. J. Tyson, and T. M. Murali. Top-down network analysis to drive bottom-up modeling of physiological processes. Journal of computational biology : a journal of computational molecular cell biology, 20(5):409-18, may 2013.

[4] A. Ritz, C. L. Poirel, A. N. Tegge, N. Sharp, K. Simmons, A. Powell, S. D. Kale, and T. Murali. Pathways on demand: automated reconstruction of human signaling networks. npj Systems Biology and Applications, 2:16002, mar 2016.

[5] S. D. Schlatterer, M. A. Tremblay, C. M. Acker, and P. Davies. Neuronal c-Abl overexpression leads to neuronal loss and neuroinflammation in the mouse forebrain. Journal of Alzheimer's disease : JAD, 25(1):119-33, jan 2011. 